\section{Mice and men measure up}

Mice have long been a favourite model for understanding human genetics and disease. But just how similar are mice and humans? Now that the genome sequences of both species are almost complete, the comparative genomics approach looks like being the most propitious means to answer this age-old question. Mural et al., from Celera, Inc., have been the first to try it out on a large scale. By comparing the structure and protein-coding potential of mouse chromosome (MMU) 16 with human genome sequence, they have shown that large blocks of MMU 16 are shared between these two mammals. In addition, $\sim 95 \%$ of annotated genes on MMU 16 are also present in humans, some of which are homologues of human disease genes. We now have a good idea of how similar the mouse and human genomes are, and how evolution has shaped them in the 100 million years since they diverged.

MMU 16 was extracted from the draft sequence of the mouse genome that the authors obtained using the whole-genome shotgun method - the same approach used by Celera to sequence the fly and human genomes. So what do we see when the MMU 16 sequence, which has $5.3 \times$ coverage and is freely available in GenBank, is compared to the human genome? What stands out first of all are long stretches of chromosome that contain homologous genes. Although these chunks are reorganized relative to each other, the linear order of genes within each block is surprisingly well conserved. Indeed, almost all the $>11,000$ homologous DNA stretches ('syntenic anchors') found within the blocks fell in the same relative position in both species. As Neal Copeland points out in an accompanying Perspective article, this will come as good news to genome assemblers, as the conserved syntenic relationships

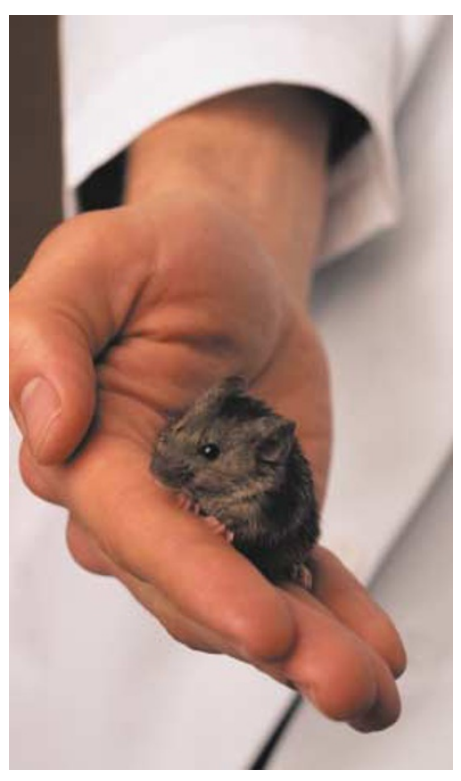

provide independent confirmation of their contig assembly schemes. Also, many syntenic anchors (44\%) lie outside genes, impelling us to find out more about what has constrained the evolution of such regions. Only 14 of the predicted 731 MMU 16 genes are not found in humans, and about two-thirds are present in positions of conserved synteny with one of the five human regions to which the MMU 16 blocks map. However, the genomes part company when it comes to tandem gene clusters, such as olfactory receptors; in these regions, duplications seem to have arisen from different founder sequences in the two mammals. Another disparity lies in the size of the two genomes - the mouse genome is $90 \%$ that of humans, mainly because it contains fewer repetitive elements, such as SINEs.

This work confirms the similarity of humans to our whiskered friends; it also allows us to speculate on how mammalian genomes were sculpted into having such a patchwork appearance (through genome rearrangement) and offers new problems to contemplate, such as why intergenic regions are so conserved. These and other mysteries lie ahead, some of which might only be resolved once more mammalian genomes have been added to the growing list of sequenced metazoans.

\section{9) References and links}

Tanita Casci ORIGINAL RESEARCH PAPER Mural, R. J. et A comparison of whole-genome shotgun-derived mouse chromosome 16 and the human genome. Science 296, 1661-1671 (2002)

FURTHER READING Copeland $\mathrm{N}$ G et al Mmu 16 RTHER READing Cop

\section{IN BRIEF}

\section{POPULATION GENETICS}

Founding mothers of Jewish communities: geographically separated Jewish groups were independently founded by very few female ancestors.

Thomas, M. G. et al. Am. J. Hum Genet. 70, 1411-1420 (2002)

Thomas et al. compared the maternal and paternal genetic histories of nine Jewish communities with those of the population among whom the groups have lived for many generations. Their data indicate that most groups were founded, independently, by few women, with little female genetic input from the surrounding community. However, Y-chromosome diversity bears similarities to the host population, highlighting how cultural practices here, female-defined ethnicity — influence genetic variation.

\section{MOUSE MODELS}

A long CAG repeat in the mouse Sca1 locus replicates SCA1 features and reveals the impact of protein solubility on selective neurodegeneration.

Watase, K. et al. Neuron 34, 905-919 (2002)

Watase et al. have produced a new mouse model of the human, polyglutamine neurodegenerative disorder, spinocerebellar ataxia type 1 (SCA1), by targeting mouse Sca1 with a mutant Sca1 $\left(\right.$ Scal $\left.{ }^{154 Q / 2 Q}\right)$ gene that contains 154 CAG repeats. Mutant mice reproduce many aspects of human SCA1, but, interestingly, mutant protein aggregates do not form in neurons most affected in SCA1 until late in the disease, raising further questions about the role of these aggregates in SCA1 pathology.

\section{GENETIC NETWORKS}

Robustness, flexibility, and the role of lateral inhibition in the neurogenic network.

Meir, E. et al. Curr. Biol. 12, 778-786 (2002)

Having generated a computer model of the genetic interactions that operate in fly segmentation, the authors now test whether the same robust properties apply to the neurogenic network. The model successfully shows three typically neurogenic patterning behaviours, including lateral inhibition. Importantly, the modelled network can perform these behaviours over a wide range of parameter values. Such robustness to noise could afford the system the evolutionary flexibility to explore new developmental patterns.

\section{GENOMICS}

\section{A global analysis of Caenorhabditis elegans operons.}

Blumenthal, T. et al. Nature 417, 851-854 (2002)

Unlike most animals, C. elegans has operons. Previous evidence indicated that the processing of polycistronic operon transcripts involved transplicing of individual ORFs to an SL2 leader. Blumenthal et al. confirm this finding by using microarrays to screen the worm genome for SL2. They report that SL2 is a hallmark of within-operon transplicing and that the worm has at least 1,000 operons that comprise $\sim 15 \%$ of its genes. 